\title{
Philosophiques
}

\section{Commentaire sur L'inquitétude de la pensée}

\section{Isabelle Thomas Fogiel}

Volume 44, numéro 2, automne 2017

URI : https://id.erudit.org/iderudit/1042337ar

DOI : https://doi.org/10.7202/1042337ar

Aller au sommaire du numéro

Éditeur(s)

Société de philosophie du Québec

ISSN

0316-2923 (imprimé)

1492-1391 (numérique)

Découvrir la revue

Citer ce document

Thomas Fogiel, I. (2017). Commentaire sur L'inquitétude de la pensée.

Philosophiques, 44(2), 327-333. https://doi.org/10.7202/1042337ar d'utilisation que vous pouvez consulter en ligne.

https://apropos.erudit.org/fr/usagers/politique-dutilisation/ 


\title{
Commentaire sur L'inquitétude de la pensée
}

\author{
ISABELLE THOMAS FOGIEL
}

Département de philosophie

Université d'Ottawa

Tout d'abord, dire combien ce livre me semble important et marque une étape décisive dans la réception de la pensée de Heidegger. Certes, je n'en suis pas spécialiste et l'on pourrait dès lors en conclure que ce jugement enthousiaste ne reflète que les limites de son auteur. Pour désamorcer ce soupçon, qui voilerait injustement l'événement que représente objectivement ce livre, sans doute convient-il de rappeler la situation dans laquelle se trouvaient, en France, avant sa parution, les professeurs de philosophie. Ils étaient confrontés à trois positions qui se partageaient la scène des études heideggériennes françaises. La première était clairement celle des disciples qui, dans le sillage de J. Beaufret, commentaient la parole du maître, reprenant ses principaux motifs en vue de les porter toujours plus haut, plus loin. Cette ferveur a commencé à se tarir au cours des années 80 , où il me semble que peu d'étudiants, qui entamaient leurs études de philosophie au moment de la disparition de Beaufret, ont souhaité embrasser la geste heideggérienne des générations précédentes. C'est ainsi qu'à la ferveur a succédé la dénonciation. Elle fut marquée par les différentes "affaires Heidegger» dans les années 90, qui connurent leur acmé en 2005 avec le livre d'E. Faye, travail qui aujourd'hui encore produit d'importantes ramifications. Entre ces deux pôles antinomiques se tenaient de grands historiens de la philosophie (tel Courtine), qui, au rythme de la publication des œuvres complètes, élucidaient les textes, accumulaient les exégèses, souhaitant conférer à Heidegger sa stature de "classique ", au côté d'Aristote, Descartes et Kant. Cependant, bon nombre de ces spécialistes, passés par l'enseignement de Beaufret (tels Courtine, Martineau, et une grande partie des protagonistes interrogés par Janicaud, dans son Heidegger en France), étaient enclins à épouser, dans des interventions autres que de commentaires, un certain nombre de décisions heideggériennes (l'histoire de la métaphysique comme onto-théologie, la critique de la philosophie dite de la subjectivité, voire celle de la raison). Cette orientation semblait, parfois, aller à l'encontre de leur volonté de faire de Heidegger un classique, tout se passant comme si (du moins pour l'observateur extérieur) être spécialiste de Heidegger devait signifier être heideggérien. C'est ce paysage français que le livre sur le jeune Heidegger de Sophie Jan Arrien (à l'avenir SJA) a profondément modifié. Certes, Greisch, en 1994, avait déjà balisé les principales étapes de la genèse d'Être et Temps; certes, les études en français sur le jeune Heidegger n'étaient plus si rares depuis l'ouvrage dirigé par Courtine en 1996. Mais outre le fait que ces études sur l'avant Être et Temps étaient toutes vectorisées par le souci d'ex- 
pliquer l'ouvrage de 1927, elles prenaient souvent l'allure de coup de sonde sur tel ou tel aspect particulier (impression accentuée par la forme de succession d'articles dans des ouvrages collectifs).

Le parti pris par SJA sur le jeune Heidegger (1919-1923) est tout autre: d'une part, elle refuse de le lire à l'ombre d'Etre et Temps. Elle considère cette période de Fribourg comme une totalité organique, signifiante par soi, sans la rapporter à la problématique ultérieure de l'Être, qui ne s'amorça véritablement, comme l'avait montré Greisch, qu'en 1924. D'autre part, elle restitue la cohérence du parcours à partir de l'exigence principielle du jeune Heidegger : lier indissolublement la conceptualité philosophique à la labilité de la vie. Elle montre pourquoi cette tâche, loin de refléter un thème assurément dominant de l'époque (celui de la vie), trouve sa teneur particulière et son originalité propre dans ces cours de Fribourg. Ce faisant, elle décrit une philosophie en acte (et non plus en gestation), une pensée toujours inquiète qui se concrétise par un dialogue serré et une critique minutieuse de ses principaux contemporains (Rickert, Lask, Natorp, Dilthey). Enfin, elle organise les principaux thèmes ou pistes de recherche en un système (au sens neutre de ce qui est posé ensemble - sun istemi - et non jeté au hasard de fulgurances ponctuelles); composition cohérente qui montre le lien d'implication entre les motifs mis en avant par ces cours de Fribourg, à savoir: 1) le thème directeur, porteur de l'ensemble de l'édifice, d'une philosophie qui doit renoncer à toute élucidation extérieure à son objet, à toute "vision du monde ", à toute posture épistémologique pour se faire "science préthéorique originaire » (chapitre I);2) le motif de l'originaire. Cette "originarité » est antérieure à toute position de sujet et d'objet, sorte d'Ur-etwas se présentant dans un vécu mondain, lequel est indissolublement sujet et objet, et non plus expérience d'un sujet face à un monde qu'il maîtriserait du regard. Ce thème de l'originaire s'explicite comme "significativité " du monde ambiant immédiat, à partir duquel le sujet advient et tout à la fois «se reçoit» (p. 73), non pas comme sujet théorique mais comme ouverture au monde, Ereignis, facticité (chapitre II); 3) le thème de la vie comme énergie créatrice de sens, qui est "donnée ou prédonnée avant que la méthode qui doit précisément la découvrir soit explicitement mise en œuvre»(p. 145). Vie, donc, qui, semble, avec le jeune Heidegger, devoir maintenant porter les attributs anciennement assignés à l'Ego: se dire soi-même par soi-même, revenir sur soi, auto-suffisance et auto-appartenance (chapitre III); 4) le thème de la destruction (chapitre IV et V), qui permet la mise en œuvre d'une méthode qui vise, à partir de la déconstruction des concepts traditionnels qui recouvrent et occultent "la vie", à retrouver leur source originaire. Méthode qui passera par la mise au jour de la dimension de praxis vécue, d'effectuation concrète que porte la notion "d'indication formelle ", que SJA propose d'écrire concept, marquant ainsi la distance entre la saisie purement théorique (le «capere» ou la «prise» du concept, qui abstrait, 
fige et mutile) et la dimension d'effectuation, de performance propre au comprendre originaire de la vie, proposé par Heidegger.

Tous ces thèmes sont articulés entre eux, et SJA en restitue les liens d'implication, en dévoile la dynamique logique, qui font de cette première philosophie de Heidegger une totalité qui se suffit à elle-même, et dont SJA semble dire, dans sa conclusion, qu'elle portait peut-être plus de promesses que la thématique de l'être n'en a par la suite accomplie.

La démonstration de SJA est exigeante, rigoureuse et sans complaisance. Chaque nouvel énoncé de Heidegger est introduit par des questions directes, qu'en une autre époque de la réception du philosophe, on eût jugé triviales, telles: «que signifie X (le vécu, l'originarité) ou «mais comment peut-il affirmer $Y$ ?». Elle anticipe toujours les éventuelles réserves du lecteur, plutôt que d'imiter le style parfois assertorique, sinon oraculaire, de certains écrits du maître. Bref, qu'il soit spécialiste ou non, le lecteur a clairement la possibilité à la fois de comprendre en profondeur les questions que se pose Heidegger et d'évaluer les décisions qui en découlent. Il y gagne la possibilité de pouvoir disputer de ces options. Par là, la philosophie de Heidegger, devient, comme toute autre, une pensée interrogeable mais aussi critiquable philosophiquement. C'est pourquoi il ne me semble pas excessif de dire que ce livre réintègre Heidegger dans le débat philosophique, là où le lecteur, qui n'avait pas consacré sa vie entière à cet auteur (tel un moine médiéval à la Bible), semblait condamné à être balloté entre gloses mimétiques et attaques violentes, ou alors astreint, étant donné la série d'articles consacrés à l'auteur, à telle analyse d'un thème particulier (Heidegger et la technique, Heidegger et la métaphysique, etc.), sans qu'une reconstitution de l'enchaînement des propositions au sein d'un corpus soigneusement délimité ne lui fasse comprendre la nécessité de telles ou telles options principielles. Ce livre annonce donc que le temps est venu, pour tous, de se confronter sereinement à cette philosophie. Libérer la possibilité de discuter de la philosophie de Heidegger sans en être ni l'adepte ni le dénonciateur, tel est l'effet insigne du grand travail de SJA.

Cela précisé, mon intervention aurait pu consister à discuter en son détail tel ou tel motif particulier. Par exemple, le rapport à Natorp, dont il peut parfois sembler qu'il est moins de rupture et de dépassement que SJA, suivant Heidegger, semble parfois le conclure. Par exemple, encore, le rapport, à bien des égards troublants, de Heidegger à la conceptualité fichtéenne (via Lask, évidemment), dont on peut étonnamment retrouver des motifs, non pas seulement quant à la nécessité pour la philosophie de rendre compte de sa propre conceptualité et de ses propres conditions (aspect magistralement analysé dans le chapitre I), mais aussi quant à la notion «d'indication formelle» qui, dans la pensée, privilégie le faire, la praxis et "l'effectuation" (maître mot de Fichte pour parler du concept lui-même). Heidegger, dans cette percée, a-t-il vraiment institué une dimension nouvelle, qui lui aurait été uniquement inspirée par sa méditation de penseurs 
religieux (Paul, Augustin, Luther, chapitre V), ou bien retrouve-t-il (via Lask, encore) une des dimensions les plus marquantes de Fichte (l'association de la pensée et de l'effectuation)? Par exemple, enfin, le rapport de Heidegger (via Lask toujours) à l'idéalisme allemand, à travers le thème directeur de la nécessité du dépassement de la scission sujet/objet, exigence qui, on s'en souvient, était celle du plus ancien programme de l'idéalisme allemand. Mais ces questions qui toutes convergent vers une interrogation plus générale: dans quelle mesure Heidegger transfère-t-il au «domaine préthéorique» une partie de la conceptualité héritée de l'idéalisme allemand? ne sont peut-être pas les plus urgentes. Aussi irai-je au plus général, à savoir le lien d'implication entre les thèmes centraux, qui, à mon sens, sont: la destruction de la conceptualité philosophique, la dérivation du théorique à partir du préthéorique, et la centralité de la thématique de «l'originarité ».

Heidegger reprend l'idée de «science» et l'exigence qu'elle implique de ne pas faire de présuppositions indues (voir Husserl). Il reprochera ainsi à Rickert (et par delà lui, à toute la philosophie) de présupposer, comme un factum, la valeur absolue de la vérité, alors qu'il faudrait la questionner (pourquoi vouloir le vrai ?, demandait déjà Nietzsche). Aussi une des tâches de SJA est-elle de restituer la manière dont Heidegger, à chaque étape de son cheminement, montre la nécessité d'introduire tels thèmes ou d'effectuer telles percées (comme la destruction, l'originarité, etc.). Montrer la nécessité consiste à démontrer qu'il n'y a aucune autre option possible. On le fait en dévoilant les apories inextricables en lesquelles ont sombré les philosophes contemporains, confrontés au même problème: saisir philosophiquement la vie. C'est ainsi que chacun des chapitres de SJA, qui élucide les motifs nouveaux introduits par Heidegger, se consacre également à un contemporain critiqué par Heidegger (chapitre I: Rickert et Lask; II : Natorp et Husserl; III: Dilthey; IV: à nouveau Natorp et Dilthey). Ce qui, dit positivement, signifie simplement: il n'y a pas de solution de rechange à la destruction, qui elle-même est liée à la nécessité de dériver le théorique du préthéorique, lequel est à la fois le but de la philosophie et ce dont elle procède. Dès lors, retrouver l'originaire (que nous délivre le préthéorique) est ce qui doit guider l'élaboration philosophique.

C'est cette succession d'implications qui me pose encore un problème.

Je prendrai donc chacun de ces thèmes en montrant comment une autre possibilité semblait offerte. Si tel est bien le cas, ces thèmes du jeune Heidegger pourraient apparaître comme des présuppositions massives. De ces éventuelles présuppositions, SJA n'est évidemment pas comptable, car de quel philosophe - Husserl et Hegel compris — peut-on dire qu'il ne présuppose rien? Néanmoins, ces postulats, une fois éclairés, pourraient nous délivrer un biais pour comprendre le passage à Etre et Temps, dont le livre de SJA, à force de vouloir faire de la période de Fribourg une totalité organique, a peut-être transformé en véritable énigme. Pourquoi Heidegger a-til, ensuite, emprunté d'autres voies? Ou même a-t-il vraiment emprunté 
d'autres chemins, même s'il peut sembler évident que passer de la "vie " à «l'Être » modifie considérablement les analyses? Doit-on vraiment considérer le parcours de Heidegger comme une série de tournants (Fribourg, Marbourg puis après Etre et Temps, la fameuse Kehre) ? N'est-ce pas plutôt le déploiement constant de trois gestes structurels (ou, si l'on préfère: intuition nodale) qui confère à l'ensemble de sa philosophie sa coloration spécifique? Telles sont les questions naïves que je me suis posées grâce à la lecture de ce livre et sur lesquelles je souhaiterais entendre son auteur.

Reprenons donc chacun des thèmes: 1) destruction, 2) dérivation, 3) origine.

1) Ce thème, traité frontalement par SJA dans les derniers chapitres, apparaît néanmoins dès le chapitre I comme une motivation germinale. Dès les cours de 1919, il se donne comme souci directeur lié à la nécessité de penser la vie. Néanmoins, l'on peut se demander quel est le lien intrinsèque entre le souci de faire droit au "vécu facticiel et historique " et l'entreprise de destruction de la conceptualité philosophique traditionnelle. En effet, en admettant la critique que Heidegger fait de Rickert, et de toute la philosophie, on aurait pu concevoir qu'il en conclut que la philosophie est restée cantonnée au sujet de la connaissance et qu'elle doit maintenant s'étendre à d'autres domaines, et, pour ce faire, inventer de nouveaux outils. Pour le dire autrement, la conclusion, tirée du diagnostic relatif à la restriction dommageable de la philosophie à la seule dimension théorique de l'existence humaine, aurait pu être la proposition d'une extension de la philosophie à des domaines qu'elle avait jusqu'alors délaissés. Or «étendre " ne veut pas dire «détruire» ou «faire exploser» le passé, mais intégrer les niveaux anciens dans un espace devenu plus large. Aborder les thèmes de la facticité, du vécu, de l'événement, etc., n'implique donc pas de faire table rase de toute la conceptualité philosophique antérieure. Cette question: "Pourquoi détruire plutôt qu'étendre le domaine de la philosophie? ", se pose d'autant plus à Heidegger que tous ses contemporains, partageant son diagnostic d'une insuffisance de la conceptualité philosophique, ont choisi l'autre option. Husserl souhaitait « recommencer la philosophie », Natorp la « reconstruire», et Laks "l'élargir» en la confrontant à la "masse sensible du contenu ", défini comme diversité du donné et non plus simplement comme énoncé de la science. Il devient donc difficile, même en suivant la démonstration rigoureuse de SJA, de comprendre pourquoi le choix de la destruction serait un choix sans autre option possible. Le chapitre I nous indique que Lask (qui proposait un élargissement) a échoué, aux yeux de Heidegger, parce que «il ne rend pas compte de la possibilité pour le discours philosophique de se comprendre à partir de son contexte (antéprédicatif) d'origine» (p.60.) La notion de «destruction» semble donc induite par ce motif de la nécessité d'une dérivation du théorique à partir de l'antéprédicatif ou préthéorique. Mais que le théorique doive dériver du préthéorique peut là encore sembler d'une décision initiale de Heidegger. 
2) En effet, pourquoi vouloir que le contexte théorique ait pour origine le préthéorique? Il peut sembler qu'une possibilité moins radicale que la dérivation s'offrait à la pensée. Elle consistait simplement à dire que théorique et préthéorique sont deux sphères qui ne se recouvrent pas, au sens ou l'une ne dérive pas de l'autre, mais se complètent ou, à défaut, voisinent. En effet, d'un point de vue général, nous pouvons considérer le préthéorique (comme par exemple l'expérience sensible) comme un domaine parmi d'autres, une strate ou encore une sphère qui a son logos propre. Reconnaître la pertinence d'une strate de l'expérience (occultée par la philosophie passée) ne nous contraint pas logiquement à en faire une source, un sol, un fondement dont devrait être dérivée la totalité de nos activités. Une strate, pour avoir sa légitimité, à un certain niveau, peut ne plus en avoir à un autre. On pourrait, ici, comparer l'exigence impérieuse de dérivation de Heidegger au dispositif du Husserl tardif (celui de la Krisis). En effet, non seulement chez Husserl, le "monde de la vie» ne se réduit pas au monde seulement préthéorique puisqu'il y inclut la culture scientifique, mais encore il n'est pas question de détruire la conceptualité théorique (mathématiques ou autres) à partir de l'appréhension du préthéorique. Il s'agit plutôt de différencier les différentes strates et de les situer chacune à leur niveau de pertinence. Le geste de Husserl consiste à étendre toujours plus l'investigation phénoménologique, à arpenter de nouveaux domaines, à découvrir de nouveaux lieux. Dans une telle configuration, la considération du préthéorique n'avait pas le pouvoir de détruire le théorique puisqu'il se donnait seulement comme un niveau différent que la philosophie pouvait envisager parmi d'autres strates ou composantes de notre monde.

Là encore, le choix de Heidegger apparaît moins contraint par une nécessité philosophique (sur le mode: il n'y a pas d'autres solutions), que guidé par une présupposition (une décision) dont nous peinons, parfois, à trouver la clé. Tout se passant comme si les positions du jeune Heidegger étaient constamment animées d'un parti pris de radicalité, qu'a souligné en son temps S. Mosès. Dans les deux cas (destruction/dérivation), Heidegger choisit la solution la plus radicale, la plus en rupture avec toute tradition. Pourquoi «détruire» le théorique et le «dériver» du préthéorique? Le troisième thème (celui de l'originaire) nous permet-il de le comprendre, ou bien est-ce là encore un présupposé problématique?

3) La philosophie a affaire à l'originaire. Ce qu'elle doit rechercher, c'est la source. Le geste philosophique fondamental de Heidegger me semble être de substituer à la visée de vérité celle de «l'originarité». Est recevable un concept qui a été reconduit à sa source. Il y a une origine dont tout procède, à laquelle nous devons constamment revenir, et qui, en retour, délivrera notre avenir. C'est cet Ur-etwas qui est porteur et créateur de sens. Néanmoins, face à ce thème de l'originaire, il m'a semblé que la critique qu'il adresse à la philosophie: présupposer un certain nombre de thèses (comme celle de la nécessité de chercher la vérité) sans jamais la questionner 
pourrait se retourner contre lui. Pourquoi vouloir l'originarité? De même que Nietzsche remplaçait l'exigence de vérité par celle de la santé (dimension qui elle aussi ressortissait à la thématique de la "vie»), Heidegger lui substitue la visée de l'originaire, sans que jamais ne se dissipe la question qu'ils ont eux-mêmes initiée. Pourquoi «l'idée même de philosophie comme science originaire vise[rait-elle] à rendre visible l'origine» (cours de 1919 cité par SJA p. 39) ? Le triangle (dont destruction et dérivation sont deux des côtés) a donc pour base l'originaire, sur lequel tout repose et dont tout dépend. Mais pourquoi l'origine plutôt que la vérité? En quoi l'origine en tant que telle vaudrait plus que tout autre chose?

Or il me semble que ces trois traits: destruction, dérivation et thématique de l'origine, tous emprunts d'un souci de radicalité à connotation révolutionnaire, peuvent se retrouver dans sa philosophie ultérieure. Cela étant, la rupture ou césure entre la période de Fribourg et celle d'Être et Temps (voire plus tard encore) me semble moins significative que le livre de SJA ne le laisse penser. Certes, il y a bien rupture entre l'idée même d'une ontologie (comme question de l'être) qui relativise ou «régionalise» la vie ( $\$ 10$ de $S u Z$ ), et le fil d'Ariane de la «vie». Mais est-ce que cela change les trois gestes fondamentaux qui caractérisent cette période de Fribourg? Que l'origine ou originaire soit déterminé comme «Être» ou comme «Vie» (ou comme Etwas = X) n'entame pas la nature de ces trois gestes: destruction (de la philosophie), dérivation (du théorique à partir du préthéorique), recherche obstinée d'une origine dont tout procède et à laquelle tout revient. Gestes fondamentaux qui me semblent décider de la teneur générale de toute la philosophie de Heidegger, quelle que soit la période considérée.

Quoiqu'il en soit, ces questions de non-spécialiste disent la nécessité de se confronter à «l'inquiétude de la pensée». 